\title{
Communicating new knowledge on previously reported genetic variants
}

\author{
Samuel J. Aronson, ALM, MA ${ }^{1,2}$, Eugene H. Clark, BM ${ }^{1,2}$, Matthew Varugheese, $\mathrm{MS}^{1,2}$, \\ Samantha Baxter, MS, CGC ${ }^{3}$, Lawrence J. Babb, BS ${ }^{1,2}$ and Heidi L. Rehm, PhD, FACMG S $^{3,4}$
}

Genetic tests often identify variants whose significance cannot be determined at the time they are reported. In many situations, it is critical that clinicians be informed when new information emerges on these variants. It is already extremely challenging for laboratories to provide these updates. These challenges will grow rapidly as an increasing number of clinical genetic tests are ordered and as the amount of patient DNA assayed per test expands; the challenges will need to be addressed before whole-genome sequencing is used on a widespread basis.

Information technology infrastructure can be useful in this context. We have deployed an infrastructure enabling clinicians to receive knowledge updates when a laboratory changes the classification of a variant. We have gathered statistics from this deployment regarding the frequency of both variant classification changes and the effects of these classification changes on patients. We report on the system's functionality as well as the statistics derived from its use.

Genet Med 2012:14(8):713-719

Key Words: clinical decision support; electronic health record; GeneInsight; genetic reports; knowledge base; report updating; variant classification

\section{INTRODUCTION}

Rapidly falling DNA sequencing costs are generating considerable excitement in many fields. ${ }^{1}$ In the area of clinical medicine, these cost reductions are making it economically feasible to decrease the cost of sequencing-based diagnostic tests while increasing the portion of patient DNA covered in each test. However, as sequencing technology advances there is a growing realization that geneticists, genetic counselors, physicians, and other health-care providers will face increasing challenges in managing and applying these results over time.

Laboratories that conduct sequencing-based tests currently encounter considerable challenges when delivering useful interpretive reports. These tests often identify novel genetic variants that have not been seen before. In many cases, it is not possible to interpret the significance of these variants when they are first identified and they become Variants of Unknown Significance (VUSs). These VUSs represent a large part of the challenge in providing clinically useful reports.

The identification of a VUS in a patient often makes treatment decisions difficult. For example, in the case of hypertrophic cardiomyopathy (HCM), a VUS leaves the provider unable to use that information to predict the risk for disease and sudden cardiac death in relatives or sometimes results in incorrect assumptions and treatment decisions, despite the laboratory's best efforts to write clear reports and recommendations. ${ }^{2} \mathrm{HCM}$ is a dominant disease with delayed penetrance and variable expressivity; therefore, genotype becomes critical to predict who is at risk in the family. Without genotypic information, family members may not pursue implantable cardioverterdefibrillator placement or other forms of cardiac intervention that could save their lives.

Knowledge of the significance of a VUS often evolves over time as data are accumulated through familial segregation studies, control studies, functional analyses, or other means. At some point, it becomes possible to provide more information about the significance of the variant in diagnostic reports, both to the original family and to subsequent families who may have the same variant. It is important to ensure that this new information is incorporated into future genetic test reports. It is also important to provide updated information to providers so they can revise patient care plans of previously tested patients. In the above HCM example, if a VUS is reclassified to pathogenic, it enables testing of other family members. For those testing positive, steps can be taken to reduce risk through careful monitoring, lifestyle adjustment, and sometimes implantation of an implantable cardioverter-defibrillator. For those testing negative, repetitive and costly medical evaluations for family members can be eliminated. However, to impact care this new clinical information must reach the patient's health-care provider.

\section{SCOPE OF THE CHALLENGE}

Even for today's clinical sequencing tests, maintaining and communicating this information is difficult. It is already 
challenging for laboratories to track the state of clinical knowledge surrounding each variant that can be identified by their tests. The Partners HealthCare Center for Personalized Genetic Medicine's Laboratory for Molecular Medicine (LMM) currently manages information on 10,155 unique variants known to exist in the 219 genes covered by its 183 active clinically orderable tests. The LMM uses GeneInsight $\mathrm{Lab}^{3}$ to manage its knowledge base. When a test is first established, the assayed genes are defined in the system, which loads reference sequences from National Center for Biotechnology Information. Initial variants can be loaded into the system from external sources (e.g., data downloads from dbSNP or locus-specific databases or manual entry from literature curation), but every variant is assessed by the LMM and verified by a board-certified geneticist before it is incorporated into a patient's report. Certain benign variants can be algorithmically assessed but all others are manually reviewed. Each time a new variant is identified in a patient, it is added to the knowledge base. Because the laboratory's understanding of variants increases over time, every variant is at its own stage of its knowledge life cycle. For some, the clinical implications are very well understood; others are VUSs and still others are in between, where there is some evidence of their likely clinical implications but not enough to state these implications definitively. The LMM's knowledge base includes 1,958 (19\%) clinically significant variants (pathogenic, likely pathogenic, resistant, likely resistant, responsive, likely responsive, or likely nonresponsive), 1,148 (11\%) VUS, 3,188 (31\%) incidental variants (benign or likely benign), and 3,861 (38\%) variants not yet classified (not observed during testing but reported in the literature or in online databases for the genes tested in the lab). Knowledge surrounding these variants can evolve in different ways. In addition to knowledge being generated to update the classification of a VUS, at times contradictory evidence can emerge that necessitates changing the classification of a variant that was previously thought to be well understood.

To date, there has been no clear statement as to who is responsible for staying up to date on the implications of a patient's genetic profile: current guidelines suggest in most cases that the process should rely on a combination of effort by the patient and primary care physician, with recommendations for future inquiry suggested by the medical geneticist at the time of the original consultation. ${ }^{4}$ However, in the case of novel sequence variants guidelines suggest the laboratory should make an effort to contact the treating clinician. ${ }^{5}$ Both approaches are difficult to implement. The patient and physician are not likely to be aware of variant-level advances in genetic knowledge. Furthermore, even if updated information is uncovered it is often delayed. At best, a physician or genetic counselor is likely to contact the lab for any updates only on an annual basis, yet new information might be learned about a reported variant a few weeks after it is first reported in the patient. Even if the physician calls one year later, months of potential patient benefit are lost. New information should not be delayed.

As a result of these challenges, more pressure is being placed upon clinical laboratories to initiate the process of updating reports through an amendment process. However, this process is equally challenging for laboratories that may not have robust systems to manage an update process and currently have no mechanism to receive reimbursement for such efforts.

All of these problems will expand rapidly as the total number of variants identified in the patient population grows as a result of expanding testing volume and the increasing amount of patient DNA that can be sequenced in each test. New medical infrastructure must be established to meet this challenge. The first clinical whole-genome sequencing tests have been conducted. ${ }^{6}$ These tests identify millions of variants per patientfar beyond what any single organization can curate.

To better demonstrate the scope of this challenge, we have tracked the rate of variant classification updates in just one of the areas for which our clinical laboratory has offered testing for the past six years. The example is HCM. The first clinical test for this disease was introduced in 2004 and as of July 2011 we had conducted 4,923 tests for this disease, including 2,644 probands and 2,279 family members. As of this date, our database housed 429 HCM variants classified as pathogenic or likely pathogenic, 244 as unknown significance, and 296 variants classified as likely benign or benign. An additional 503 variants are in the lab's database, having been reported in other studies, but remain unclassified because published evidence is not evaluated until a variant is observed in a patient. The table found at the following website shows the LMM's system for classifying variants according to available evidence: http://pcpgm.partners.org/sites/default/files/LMM/Resources/ LMM_VariantClassification_05.26.11.pdf.

Over the span of these six years, we have made 214 HCMspecific classification changes to previously reported variants. These changes are primarily derived from new knowledge being gained on these variants as well as some improvements to our classification process. Figure 1 shows the volume of specific class changes for HCM variants. We have grouped these changes into three levels of significance (Table 1) depending on the likelihood that the classification change would impact care of the patient or the understanding of the patient's disease. These changes in variant categories have had an impact on 756 patient reports. These numbers slightly understate the rate of classification changes because each report may be affected by multiple classification changes. And this is only one disease. The scope of this challenge is daunting to contemplate when applied to less well-developed areas of testing, to genes more polymorphic than the sarcomere genes of HCM, and to laboratories with much higher volumes than our small academic environment.

\section{INFORMATION TECHNOLOGY CAN HELP}

To address the need for more timely and effective communication of variant updates to treating clinicians, we propose the use of information technology. In May 2010, we launched a new infrastructure to help solve this dilemma. The application, called GeneInsight Clinic, represents an extension of a larger in-house-developed software package called the GeneInsight 
Variant classification changes-HCM data

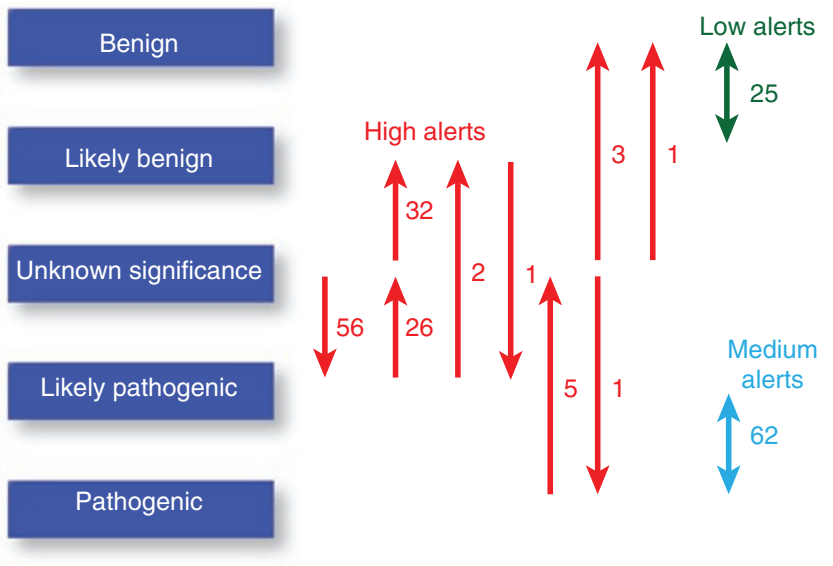

Figure 1 Variant classification changes-HCM data. Geneticist-approved variant classification changes that have occurred since 2004 on variants in 11 genes on the HCM CardioChip Test. Only changes that would have affected at least one preexisting patient report were counted. The figure also reflects only classification changes relative to HCM and does not reflect data from other diseases. These 214 changes occurred over $\sim 7$ years within our knowledge base of 1,472 HCM variants. Over this period, 4,923 tests were conducted, including 2,644 probands and 2,279 family members. HCM, hypertrophic cardiomyopathy.

Table 1 Alert categories

\begin{tabular}{ll} 
Alert categorization & Sample conditions \\
\hline High & - Variant of unknown significance reclassified \\
& to known significance \\
- Variant of known significance reclassified to & unknown significance \\
- & Benign or likely benign variant reclassified \\
& to pathogenic or likely pathogenic \\
- Pathogenic or likely pathogenic variant & reclassified to benign or likely benign \\
- New disease or pharmacogenomic & interaction associated with variant \\
& - Removal of variant association to disease or \\
& pharmacogenomic effect \\
- Likely pathogenic to pathogenic & - Pathogenic to likely pathogenic \\
- Likely benign to benign & - Benign to likely benign \\
\hline Low &
\end{tabular}

Suite, which also includes GeneInsight Lab. The GeneInsight Suite supports both germline and somatic testing processes and is deployed in multiple laboratory and provider settings across many diseases and indications for testing. The system is registered as a class I exempt medical device. A quality system based on 21 CFR 820 regulations is employed to validate the software. This reference provides additional information on the system. ${ }^{3}$ Figure 2 displays the high-level workflow GeneInsight enables. The overall goal of the GeneInsight Clinic provider interface is to give health-care providers the ability to fully manage their patient genetic profiles. We have developed functionality to support what we feel are four main functions any system must have to support the clinician in this new era of personalized medicine.

\section{Function 1: deliver reports}

This is the most basic function of a provider interface. It enables electronic communication of genetic reports. An example report can be found in the supplementary information associated with ref. 3.

\section{Function 2: enable providers to view all genetic tests that} have been conducted on a patient

Health-care providers should have immediate access to all tests that have been conducted on a patient, all clinically assessed variants identified through those tests, and the associated reports. We feel it is important that the users be able to use a screen to navigate directly to formal laboratory reports, as well as pull up additional information on variants. Figure 3 shows the screen in our current application that displays this information.

Function 3: alert providers when new clinically significant information is learned about a variant previously identified in one or more of their patients

The provider interface must manage the process of notifying providers when new information becomes available on a variant previously identified in one or more of their patients. As described above, this function is currently important for targeted sequencing-based tests that identify VUSs that may be later reclassified and other situations where new evidence causes a laboratory to change a previous classification of a variant. When information changes on a previously reported variant, we generate an "alert." We found it useful to divide alerts into three categories, high, medium, and low, as noted in Figure 1. We have chosen to send e-mail alerts to providers as soon as a high alert is raised on a patient. All other alerts are sent in summary e-mails on a periodic basis, with the frequency determined by each clinic. By default, the summaries are sent once a week and identify alerts and reports that have not yet been reviewed. Based upon user feedback, we are considering discontinuing the low alerts, which occur when a variant moves between the likely benign and benign classifications. These changes occur frequently and in our view are unlikely to impact patient management. GeneInsight Clinic currently only supports genotyping and targeted sequencing-based tests. We are currently considering how this alerting mechanism should be applied to whole-genome- and whole-exome-based testing.

The first step in managing this process involves determining what updates should be transmitted. This step can be challenging because of the need to both account for patient preferences and ensure that transmitted updates are relevant to the providers using the interface. For example, in our test for Marfan syndrome we also can uncover a variant that may be associated with increased cancer risk. Some patients may want their cancer risk to be assessed and others may not. Furthermore, some physicians ordering Marfan testing may be comfortable 


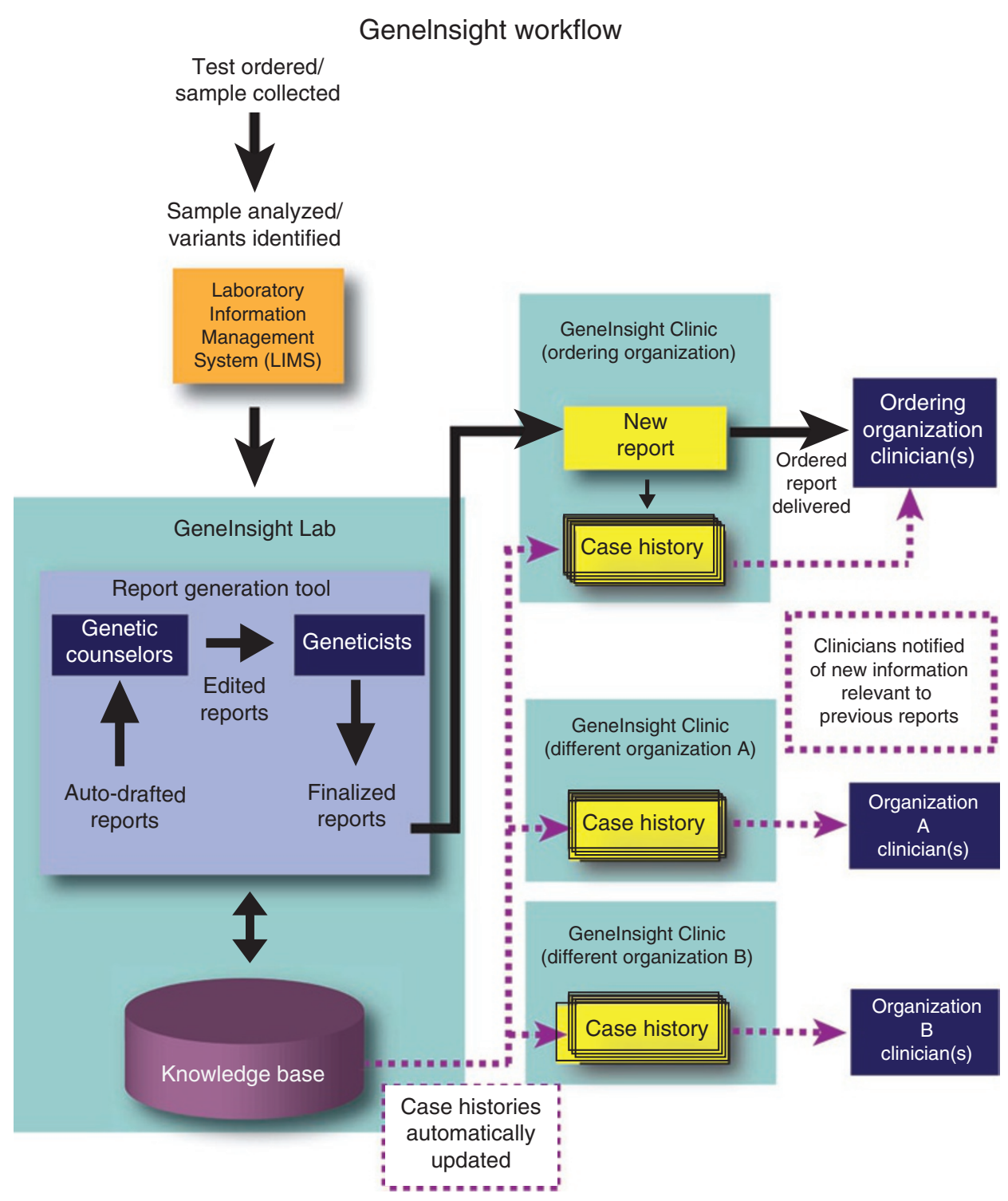

Figure 2 Genelnsight workflow. This figure depicts the high-level workflow enabled by Genelnsight. The solid black arrows represent the core reporting workflow that occurs each time a test is ordered and a sample received. The dashed arrows show an entirely automated ancillary workflow that occurs when the category of a variant is changed. This typically occurs in the course of reporting a test but can also occur independent of a test order. As a high-level view, this figure does not represent all system-level communication that occurs during the reporting process.

receiving cancer risk information and others may not be. Our current approach to managing this issue involves establishing an "interpretive scope" for each test when it is reported through the laboratory infrastructure. This interpretative scope consists of a list of diseases and/or pharmacogenomic effects that should be considered in both report drafting and future alert generation. In practice, our infrastructure is set up to generate a default interpreted scope to include only the disease areas associated with the intended use of the test. It is rare for the laboratory to have to adjust the default interpretive scope; however, this can occur in Marfan testing. Our Marfan test requisition form provides a box that can be checked to indicate whether the TGF $\beta R 1^{*} 6 A$ variant, which may be associated with cancer risk, should be included in the report. If the provider indicates that he or she would like the patient's cancer risk to be assessed, the laboratory adds this information to the test's default interpretative scope. The provider interface then respects this scope in determining whether future alerts on this variant, or other variants that could be reinterpreted later, should be transmitted. This basic approach will need to be further generalized as the scope of the average genetic test expands and patients are confronted with a wider range of questions regarding what types of genetic information they may or may not wish to receive. Solutions focused on enabling patients to initially define and later update their preferences as to what types of genetic information they wish to receive (e.g., carrier status for recessive disorders, risk for treatable late-onset disorders) have been proposed. ${ }^{7}$ 


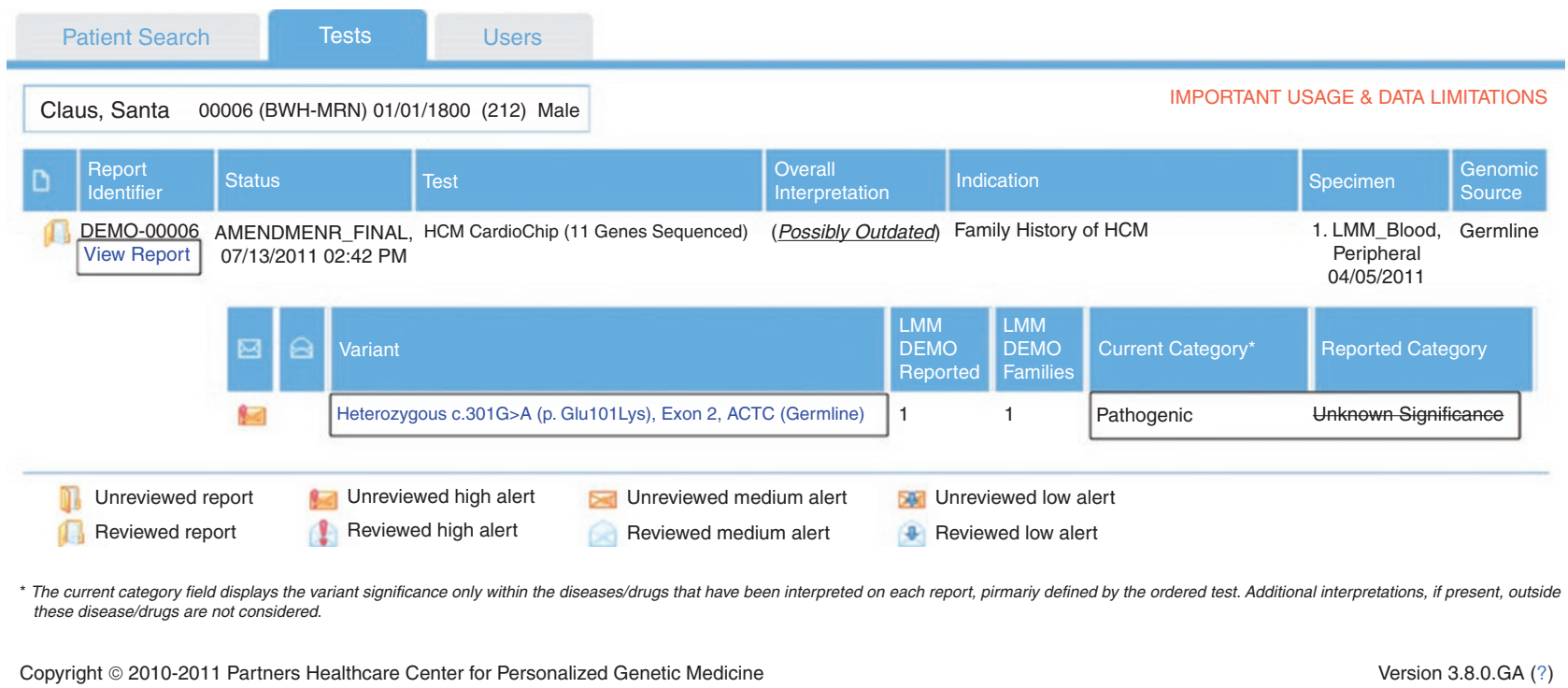

Figure 3 The Genelnsight Clinic interface. This interface image provides an example of how a screen can be assembled to manage clinical genetic test results. Users can navigate to this screen either by searching for a patient or by clicking patient-specific links in alert e-mails. (These e-mails contain no protected health information.) From this screen, users can pull up the report associated with each case or obtain additional information when clicking on a specific variant. They can also see whether the laboratory has reclassified a variant since the test was originally reported. Boxes have been added for emphasis and do not appear on the actual screen. (Adapted from ref. 3.)

When we launch a new GeneInsight Clinic, the system analyzes past reports and generates alerts for each reported variant whose classification has changed. This can result in a material number of alerts. We calculated a launch-time alert rate for each clinic by dividing the number of medium and high alerts generated at launch by the number of cases initially loaded into the clinic. Rates ranged from $3.9 \%$ to $11.2 \%$ of cases. However, after the initial launch is complete, the alert frequency slows considerably. To calculate a monthly alert rate, we began by summing the total number of months each report resided in the system. We then determined the total number of high and medium alerts generated on these reports after the GeneInsight Clinics were launched. Dividing the total post launch alerts by the number of "report months" generated an average monthly alert rate of $0.33 \%$. As tests mature, the variant knowledge becomes increasingly stable and the alert rate decreases. However, the need for alerting processes is never eliminated. Currently, $17 \%$ of clinically significant variants we identify in HCM patients are novel even after we have tested over 2,000 probands across 6 years. As a result, a large pool of variants continues to be ripe for new knowledge gains. In addition, when new DNA regions are added to a test, the knowledge accumulation process begins anew.

We have found it important to provide supporting evidence for the classification changes that generate these alerts. In our case, this evidence is entered into the text description of the variant that is used in the normal reporting system. GeneInsight Clinic applications are connected to reporting laboratory GeneInsight Lab applications. The laboratory infrastructure contains a knowledge base that tracks variant classifications relative to diseases or pharmacogenomic effects. When this variant text is updated in the GeneInsight Lab system, it is then automatically incorporated into each patient's genetic profile within the treating clinician's GeneInsight Clinic application. The system allows laboratory staff (e.g., genetic counselors, fellows, residents) to propose changes to these classifications. These proposed changes are then approved or rejected by users with appropriate permissions, typically geneticists or medical directors. Approved changes are then automatically sent to connected GeneInsight Clinic applications that generate patient-specific alerts to clinicians.

The system provides updated information through e-mail notifications and screen updates. E-mail notifications do not contain patient-specific information but rather contain links that direct the user to the patient's screen after logging in to the application. New reports are not issued because this would require geneticists to sign out a revised report for each affected patient. Our goal has been to make the update process economically feasible in an environment where these updates are not reimbursed. Because alerts are triggered when geneticists update variant-level information, usually as a result of analysis done in the course of reporting a case, generating updates often does not require additional laboratory effort. This infrastructure's effect on clinical workflow and usability is currently being studied through a National Library of Medicine grant (R1LM010526). Thus far, clinicians have not requested amended reports in response to updates. This was a welcome development. Although the LMM is able to generate amended reports for a limited number of cases, we would not have the resources to provide amended reports without charge each time a knowledge update is issued. Clinician users have provided us with feedback indicating that the system provides them with useful and timely information critical to the care of their patients. 
There is always the risk that an alert will be generated for a patient who is no longer being seen by the clinic. To date, we are only aware of two cases in which this situation has arisen. The system has been deployed to clinics associated with academic medical centers that likely have more stable patient populations than typical clinics. This may be a larger issue for community practices. Ultimately, standards and systems for addressing this issue will be required. Electronic Health Record (EHR) integrations may be able to assist if EHRs develop functionality to maintain associations between clinicians and patients. Geneticists and treating clinicians have also suggested that we could enable patients to specify an e-mail address where they can be contacted if they are no longer being seen by the ordering clinician.

Function 4: allow providers a means of searching for patients, within their patient population, who harbor a particular variant

At times a provider may decide, independent of systemgenerated alerts, to review the care of all patients who harbor a certain variant or set of variants. This may occur if they become aware of a clinical association independent of the reporting lab. This situation can be supported by providing clinicians a robust means of searching their patient population for patients that harbor the variant.

This functionality also has powerful research uses. There has been some concern expressed that clinicians could easily use the system for research purposes without proper approval. To protect against this situation, by default we restrict search results to patients for whom the user is listed as an ordering provider on the report. If the user desires to search for reports for which they are not listed as an ordering provider, he or she must supply a reason for doing the search. If the user specifies that the search is being done for research purposes, he or she must enter an institutional review board protocol number and approval date. All searches are logged and therefore auditable. Every provider organization has its own database schema containing only its own patient records, so searches cannot return another institution's records.

There are situations in which the above search restrictions could impede legitimate clinical searches. For example, when clinicians are covering for one another they may need to access reports that they did not order. In these cases, we allow the user to specify that he or she is doing an expanded search for clinical purposes.

\section{RESULTS SEEN THROUGH CURRENT DEPLOYMENTS}

GeneInsight Clinic has been deployed to a total of five clinics thus far: Brigham and Women's Hospital Cardiology, Massachusetts General Hospital Cardiology, and Massachusetts General Hospital Genetics (Boston, MA); University of Michigan Cardiology (Ann Arbor, MI); and the Fred A. Litwin \& Family Centre for Clinical Genetics and Genomic Medicine (Toronto, Canada). These clinics are currently capable of receiving reports and alerts from the Partners HealthCare Center for
Genetic Medicine's LMM. More clinics are being established. We are also working to establish connections that will allow other GeneInsight Lab users to send results into GeneInsight Clinics as well.

As of 16 August 2011, the deployed GeneInsight Clinic applications were managing a total of 2,174 reports. A total of 321 alerts on 254 patients had been sent. (More than 1 alert was issued for some patients.) Of these alerts, 124 were high alerts and 57 were medium alerts. As noted above, these alerts provide potentially critical new information to clinicians that is very difficult to obtain through other means. These numbers do not include alerts for information that the laboratory had already updated in the past through manual report amendments. These alerts were generated based on a relatively small number of highly targeted sequencing tests. Even so, without this type of information technology infrastructure, it would be difficult to maintain a process capable of generating and delivering these alerts without materially increasing the cost of testing.

\section{LINKING TO EHRS}

We chose to initially deploy GeneInsight Clinic as an independently hosted, Web-accessible system because of the time required to integrate with EHRs. This requires clinicians to log in to a separate system when they receive an e-mail alert. We informally surveyed several clinicians before making this decision. These clinicians indicated that the benefits of patient-specific variant classification change alerts significantly outweighed the need to use a separate genetic health record system, and they advised us to work to deploy the alerting functionality as quickly as possible. However, truly widespread deployment of this type of functionality will require EHR integration. These integrations are also needed to provide clinical decision support based on the integration of clinical and genetic data. We are currently working on integrations to both the Partners HealthCare and the Intermountain HealthCare EHRs. We would be very interested in working with other institutions interested in addressing these issues.

It will be important for the EHR environment to provide integrated clinical decision support that takes into account both the patient's clinical data and their genomic profile. Interfaces, capable of delivering structured case data, including identified variants and associated alerts, will need to be constructed to enable this support. For this reason, it is important that clinician facing genetic infrastructure maintain these data.

Clinical whole-exome and whole-genome sequencing tests have been launched and will need to be electronically supported. Initially, we will only load validated, clinically significant variants into GeneInsight Clinic. However, the clinical uses for whole-exome/genome sequence will likely grow rapidly, creating the need to load all identified variants, along with appropriate quality metrics, into the system. The hardware requirements associated with scaling the infrastructure in this way are currently unclear. We anticipate that only variants and quality scores will be loaded into the system, whereas all raw and intermediate supporting data will be retained by the 
reporting laboratory systems, to the extent they are retained at all. This will reduce the GeneInsight Clinic resource consumption but the database infrastructure required to store, crossindex, and back up roughly 3 million variants per patient will still be significant.

\section{CONCLUSION}

As a growing number of sequencing tests are ordered and the average number of base pairs sequenced per test increases, the number of VUSs identified in the patient population will grow rapidly. The need for infrastructure to keep providers up to date on the meaning of these variants will become increasingly critical. However, it takes time to establish interorganizational information technology infrastructure. Given the speed at which the molecular diagnostic testing industry is evolving, we must start setting up the required information infrastructure now. If we delay, the lack of information technology support may ultimately constrain the growth of molecular diagnostic testing and the widespread adoption of personalized medicine.

\section{ACKNOWLEDGMENTS}

We acknowledge all of our colleagues at the Partners HealthCare Center for Personalized Genetic Medicine who contributed to developing, testing, and rolling out the Genelnsight Suite, the Genelnsight Suite user community, which provided constant feedback, and the Refining IT Support for Genetics in Medicine team for its guidance. This work was supported in part by Partners HealthCare and National Institutes of Health (NIH) Challenge Grant

RC1LM010526 administered through the National Library of Medicine. Genelnsight Lab licensing fees also supported this effort.

The content is solely the responsibility of the authors and does not necessarily represent the official views of the National Library of Medicine or the $\mathrm{NIH}$.

\section{DISCLOSURE}

The Genelnsight Suite is made available on a hosted basis to outside laboratories. There is a fee associated with this service.

\section{REFERENCES}

1. Lander ES. Initial impact of the sequencing of the human genome. Nature 2011;470:187-197.

2. Caleshu C, Day S, Rehm HL, Baxter S. Use and interpretation of genetic tests in cardiovascular genetics. Heart 2010;96:1669-1675.

3. Aronson SJ, Clark EH, Babb LJ, et al. The Genelnsight Suite: a platform to support laboratory and provider use of DNA-based genetic testing. Hum Mutat 2011;32:532-536

4. Hirschhorn K, Fleisher LD, Godmilow L, et al. Duty to re-contact. Genet Med 1999;1:171-172.

5. Richards CS, Bale S, Bellissimo DB, et al. ACMG recommendations for standards for interpretation and reporting of sequence variations: Revisions 2007. Genet Med 2008;10:294-300.

6. Worthey EA, Mayer AN, Syverson GD, et al. Making a definitive diagnosis: successful clinical application of whole exome sequencing in a child with intractable inflammatory bowel disease. Genet Med 2011;13:255-262.

7. Kohane IS, Taylor PL. Multidimensional results reporting to participants in genomic studies: getting it right. Sci Trans/ Med 2010;2:37cm19.

(c)

बस:झाls:

This work is Commons Share Alike 3.0 Unported License. To view a copy of this license, visit http://creativecommons.org/licenses/ by-nc-sa/3.0/ 